\title{
A Comparison of DC and Modulated Photocurrent in Chalcogenide Glasses
}

\author{
R. KAPLAN* AND B. KAPLAN \\ Department of Mathematics and Science Education, University of Mersin, Yenisehir Campus, 33000 Mersin, Turkey
}

(Received September 16, 2017; in final form January 30, 2019)

\begin{abstract}
A comparison of DC and frequency modulated photocurrent measurements in amorphous (a-) $\mathrm{Se}_{2} \mathrm{a}_{-} \mathrm{As}_{2} \mathrm{Se}_{3}$, and $\mathrm{a}-\mathrm{As}_{2} \mathrm{Te}_{3}$ chalcogenide thin films are carried out at room temperature. Current-voltage $I-V$ characteristics are examined and series resistances are determined under dark and different light intensities. The exponent $v$ in the power-law relationship, $I_{p h} \sim G^{v}$ between generation rate, $G$, and photocurrent, $I_{p h}$, are calculated at different applied voltages and modulation frequencies. The small $v$ values are obtained for DC applied voltages $(25-500 \mathrm{~V})$, but high $v$ values are found for various modulation frequencies $(500-3950 \mathrm{~Hz})$. We report that $v$ is almost independent of applied voltage and modulation frequency within the experimental uncertainty, but it strongly depends on sample characteristics. The results are also compared in detail and interpreted for all threetype samples.
\end{abstract}

DOI: 10.12693/APhysPolA.135.332

PACS/topics: 71.23.Cq, 72.20.Jv, 73.50.Pz, 73.50.-h

\section{Introduction}

The interaction of light with atoms produces electronhole pairs inside the materials. The electron-hole pairs may recombine radiatively, e.g., photoluminescence, or may separate non-radiatively, e.g., photoconductivity [1]. Chalcogenide glasses of a-Se, a- $\mathrm{As}_{2} \mathrm{Se}_{3}$ and $\mathrm{a}-\mathrm{As}_{2} \mathrm{Te}_{3}$ have been a great interest for the technological applications such as photovoltaic cells, rectifiers, optical recording, optical fibers, xerography, memory, and switching devices [2-8]. The optical and electrical properties of chalcogenide materials depend on the structural configuration of the system. The Se in pure state has some disadvantages due to low thermal stability and low resistivity [9]. The addition of As and Te to Se creates a structural, compositional, and configurational disorder in the structure leading to changing the optical and electrical properties of the system. The semiconducting chalcogenide thin films usually show photodarkening under action of light from the region of fundamental absorption $\left(h v \sim E_{g}\right)[10]$.

A change of light-induced structural transformation in amorphous chalcogenide films is wide and attracts scientific as well as technological interest [11, 12]. Among them, a photo-induced diffusion has potential application in optoelectronics (photoresist, optical memories, optoelectronic circuits, etc.) [13]. The presence of localized states in the gap region of these materials is dominant factor for the photo-induced effects. The lone-pair character of the valence band tails leads to very high behavior under the influence of light. Any light illumination by band gap of many amorphous chalcogenide glasses

*corresponding author; e-mail: ruhikaplan@yahoo.com changes their internal and surface structure, and the optical absorption edge will have a red or blue shift [14]. A knowledge of the photo-interaction in such films is very important to understand the basic mechanism and its technological applications such as device production.

The DC and modulated photocurrent measurements have been widely used for understanding the defect states in the chalcogenide thin films. Recombination process is a key point when describing carrier transport kinetics in such films because it strongly affects the photoresponse of these films at all levels of external excitation [15]. The applied voltage and modulation frequency effects on exponent $v$ in the power-law relationship $I \sim G^{v}$ between generation rate and photocurrent give valuable informations about recombination kinetics and distribution of localized states in the band gap [16]. In the present work, we have determined the DC resistances of a-Se, a- $\mathrm{As}_{2} \mathrm{Se}_{3}$, and $\mathrm{a}-\mathrm{As}_{2} \mathrm{Te}_{3}$ thin films under dark and various illumination levels of HeNe laser. The effects of applied voltage and modulation frequency on exponent $v$ in $I_{p h} \sim G^{v}$ are examined and compared in detail for such thin films. It is also attempted to interpret the obtained experimental results. To the best of our knowlewdge, a very poor published data on evaluation of modulated-light intensity dependence of exponent $v$ exists. Therefore, our DC and modulated results provide a useful test of present models.

\section{Samples and experimental details}

The materials used in this work to produce samples are high purity (99.999\%) BDH chemicals. They are prepared specially for laboratory purposes. The samples were produced by using vacuum evaporating coating unit which is shown in Fig. 1. With this system it is possible to get a vacuum pressure lower than $10^{-5}$ Torr. Microscope slides $\left(76 \times 26 \mathrm{~mm}^{2}, 1.0 / 1.2 \mathrm{~mm}\right.$ thick $)$ were 


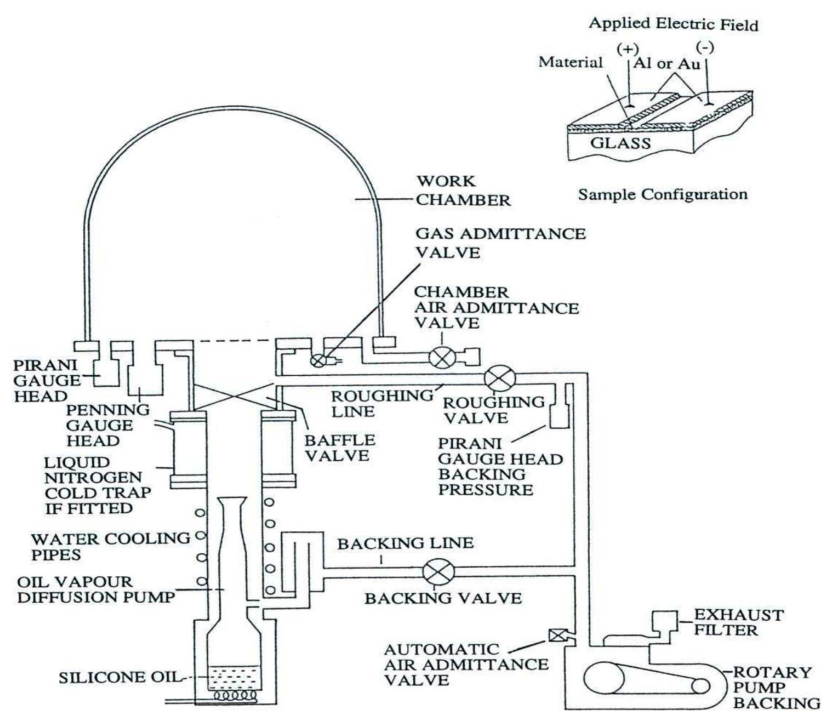

Fig. 1. Thermal evaporation unit and sample configuration used.

used as substrates. In the working chamber, materials were evaporated from a crucible (carbon, or ceramic types) surrounded by a tungsten spiral resistance (heating element) which allows a current (0-100 A) to pass through it. Many crucibles were used for each different type materials. By using a suitable $\mathrm{Al}$ foil mask, it is possible to produce many samples of the same type, in one evaporation. Evaporation sample thicknesses vary between about 0.5 and $20 \mu \mathrm{m}$. We put co-planar $\mathrm{Au}$ electrodes on the sample by using a suitable $\mathrm{Al}$ foil mask and the same evaporation unit. The electrode area was $0.5 \times 1.0 \mathrm{~cm}^{2}$. The sample geometry is roughly shown on the right side of Fig. 1. However, instead of using a crucible, the $\mathrm{Au}$ ingots were evaporated directly from tungsten spiral. For electrode spacing, the different size $\mathrm{Cu}$ wires ranging between 0.1 and $0.2 \mathrm{~mm}$ were used as masks. As in the case of depositing materials, evaporated electrodes were left to dry inside the vacuum for a while (sometimes one or two days). This is intended to prevent the oxide contamination of the sample surfaces. Copper external leads were contacted with Ag paint.

The samples were excited by a HeNe laser (Melles Griot, $1.96 \mathrm{eV}, 10 \mathrm{~mW}$ maximum). Its beam diameter $\left(1 / e^{2}\right)$ is $0.65 \mathrm{~mm}$. To obtain DC $I-V$ characteristics, an electrometer (Keithley, 6514) and a high voltage power supply (SRS Stanford Research System, Model PS325 / $2500 \mathrm{~V}-25 \mathrm{~W})$ were used for current and voltage mesurements, respectively. A chopper was used to modulate the laser light in the frequency of $500 \mathrm{~Hz}$ to $3950 \mathrm{~Hz}$. The modulated photocurrent signal excited in this way was measured and analysed by a lock-in amplifier (Stanford Research System, Model SR830). The temperature of measurement system was kept at room temperature $(295 \mathrm{~K})$. Light intensities were varied by using a set of neutral density filters.

\section{Results and discussion}

Since the energy of the excitation of light is much higher than the optical band gap of this material, we assume that the carriers are photo-excited between extended states and then a trap limited recombination occurs at room temperature.

Figure 2 shows the DC $I-V$ characteristics of a-Se thin films under dark and various levels of illumination. It gives almost straight lines for all light intensities used. This means that the samples exhibit ohmic behaviour. However, below about $100 \mathrm{~V}$ and under high illumination levels there appears a little sub-linearities. From the slopes of lines, the calculated values of series resistances $R$ are also shown in the figure. As seen, values of resistance $R$ were smaller in the light. The $R$ decreases with increasing illumination levels.

Similar results were also obtained for a- $\mathrm{As}_{2} \mathrm{Se}_{3}$ and a$\mathrm{As}_{2} \mathrm{Te}_{3}$ thin films. The dark resistances were found as 8.85, 9.79, and 5.78 $\mathrm{M} \Omega$ for a-Se, a- $\mathrm{As}_{2} \mathrm{Se}_{3}$, and $\mathrm{a}-\mathrm{As}_{2} \mathrm{Te}_{3}$, respectively. While the Te contribution to Se decreases the dark resistance, the As contribution increases it. This shows that the addition of As to Se creates much more coordination defects in structure. On the other hand, the addition of Te to Se may reduce the energy gap [10].

Under illumination, the calculated resistances versus intensities of excitations are shown in Fig. 3 for comparison. Obviously, the resistance of a-Se is larger than that of $\mathrm{a}-\mathrm{As}_{2} \mathrm{Te}_{3}$, but it is smaller than that of a- $\mathrm{As}_{2} \mathrm{Se}_{3}$ for all light intensities. Furthermore, the decrease in resistance of a-Se at low intensities $(<2 \mathrm{~mW})$ is very sharp. In general, as in the case of dark resistances, a similar trend is also observed under illumination. The DC resistance of $\mathrm{a}-\mathrm{As}_{2} \mathrm{Se}_{3}$ is almost constant with excitation

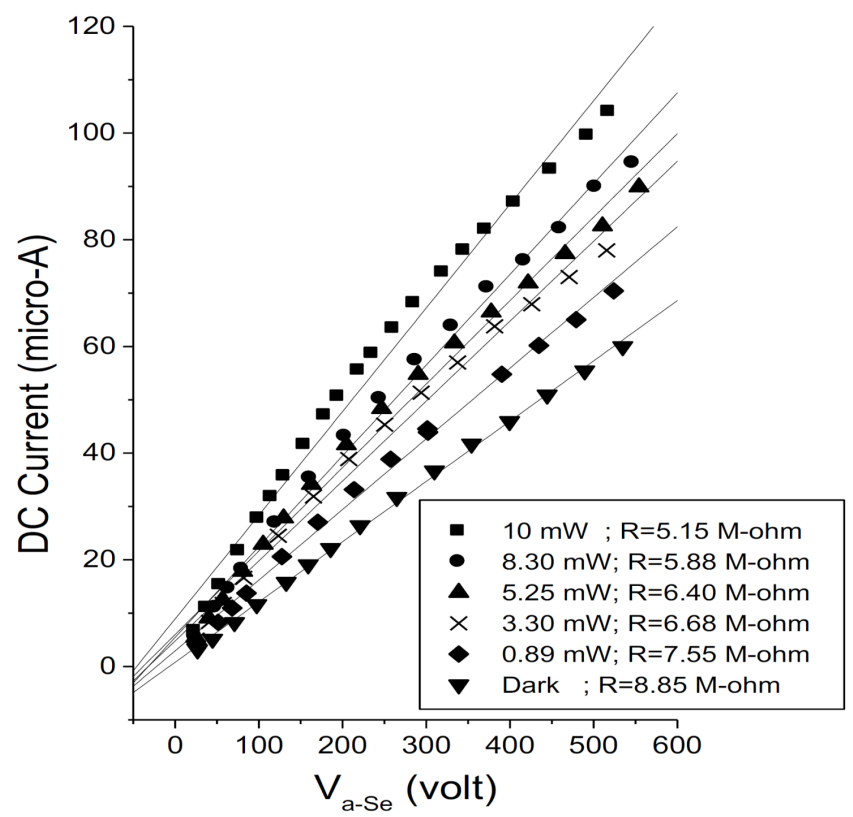

Fig. 2. DC $I-V$ characteristics of a-Se under dark and various illumination levels. 


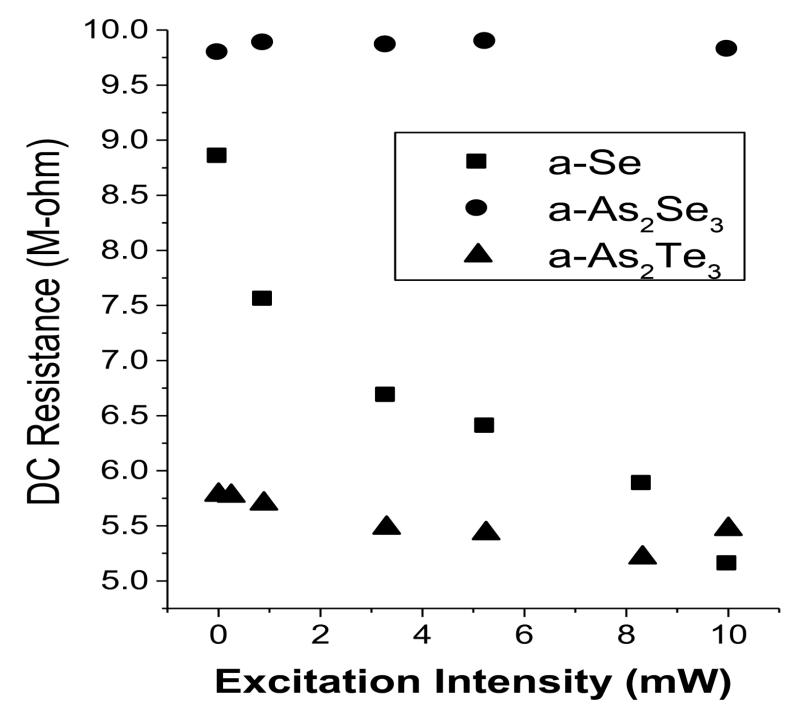

Fig. 3. Comparison of DC resistances of a-Se, a- $\mathrm{As}_{2} \mathrm{Se}_{3}$, and a- $\mathrm{As}_{2} \mathrm{Te}_{3}$ thin films as a function of excitation light intensity.

intensity. However, it decreases with increase of illumination intensity in a-Se and a- $\mathrm{As}_{2} \mathrm{Te}_{3}$ thin films. Therefore, the addition of As atom to Se structurally makes it more stable than that of Te. On the other hand, increasing the excitation intensity creates more electron-hole pairs which are separated by applied voltage (or electric field) in a-Se and $\mathrm{a}-\mathrm{As}_{2} \mathrm{Te}_{3}$, and thus their DC resistances are decreased.

Figure 4 shows the DC photocurrent of a-Se as a function of excitation light intensity at different applied voltages. As it is seen, it gives stright lines. From the slopes of lines, the calculated values of exponent $v$ in $I \sim G^{v}$ are

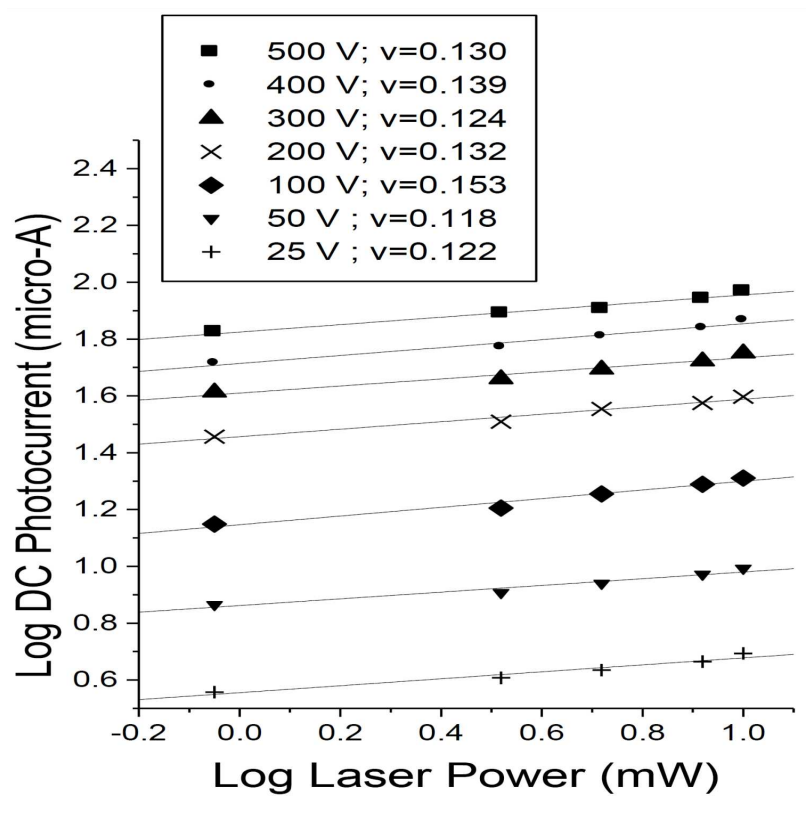

Fig. 4. DC photocurrent of a-Se as a function of excitation light intensity at different applied voltages.

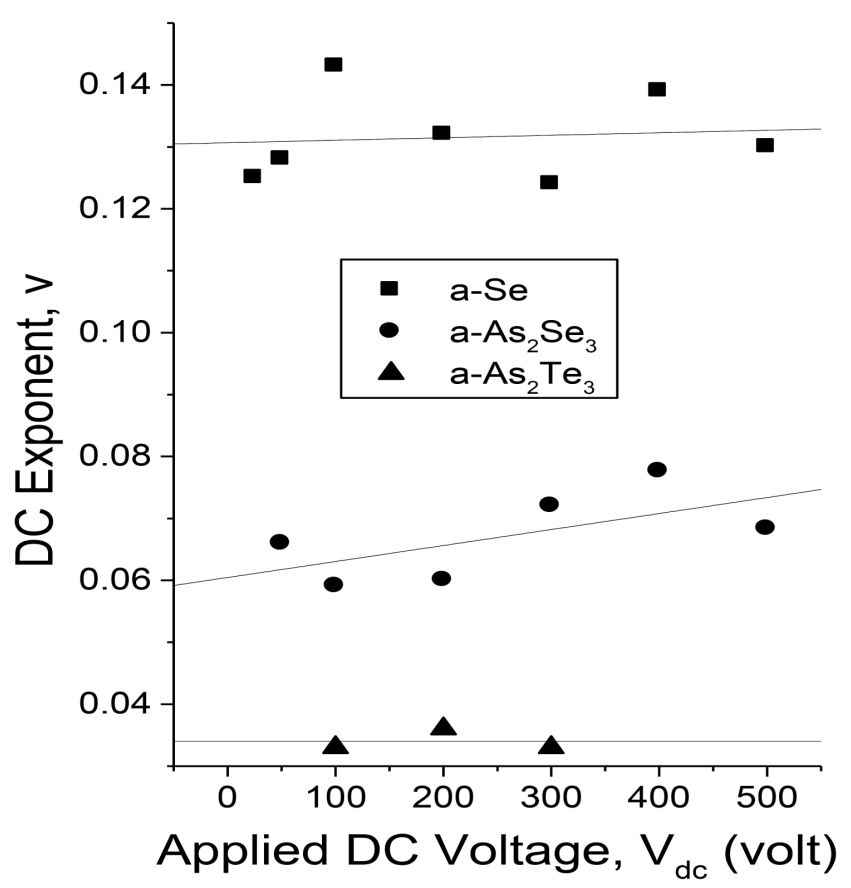

Fig. 5. Comparison of DC exponent $v$ of a-Se, a$\mathrm{As}_{2} \mathrm{Se}_{3}$, and $\mathrm{a}-\mathrm{As}_{2} \mathrm{Te}_{3}$ thin films as a function of applied voltages. Lines are drawn as guide for the eye.

also represented in the figure. The very small $v$ values are due to very high excitation light intensity used, which results in much more light-induced defects. Similar results were also obtained for other thin films of a- $\mathrm{As}_{2} \mathrm{Se}_{3}$ and $\mathrm{a}-\mathrm{As}_{2} \mathrm{Te}_{3}$. The DC exponent $v$ versus DC applied voltage are compared in Fig. 5 for all thin films worked. Obviously, the data is noisy a little bit for a-Se and a$\mathrm{As}_{2} \mathrm{Se}_{3}$, but its variation with dc applied voltage is small. The $v$ value of $\mathrm{a}_{-} \mathrm{As}_{2} \mathrm{Se}_{3}$ is larger than that of a- $\mathrm{As}_{2} \mathrm{Te}_{3}$, but smaller than that of a-Se. This may be due to their absorptions of light in energy gaps.

The dependence of photocurrent $I_{p h}$ on the photogeneration rate $G$ ( $G$ is right proportional to excitation light intensity) is given by

$$
I_{p h} \sim G^{v}
$$

where the exponent $v$ is differentially defined by:

$$
v=\mathrm{d}\left[\ln \left(I_{p h}\right)\right] / \mathrm{d}[\ln (G)] .
$$

It is now well-known that the value of $v$ differs in various chalcogenide materials [17]. Mostly, a sublinear dependence is found and the $v$ in Eq. (2) has quite complicated variation with light intensity, photon energy, modulation frequency, applied bias, and temperature. It is suggested that $v=1$ corresponds to monomolecular recombination and $v=0.5$ to bimolecular recombination. Although, in the case of continuous distribution of traps the value of $v$ may be anywhere between 0.5 and 1.0 depending on the light intensity and temperature range [15].

As seen in Fig. 5, we found a power dependence of less than 0.5 under high intensity illumination for all samples. However, this cannot be explained by conventional models of monomolecular or bimolecular recombination. 


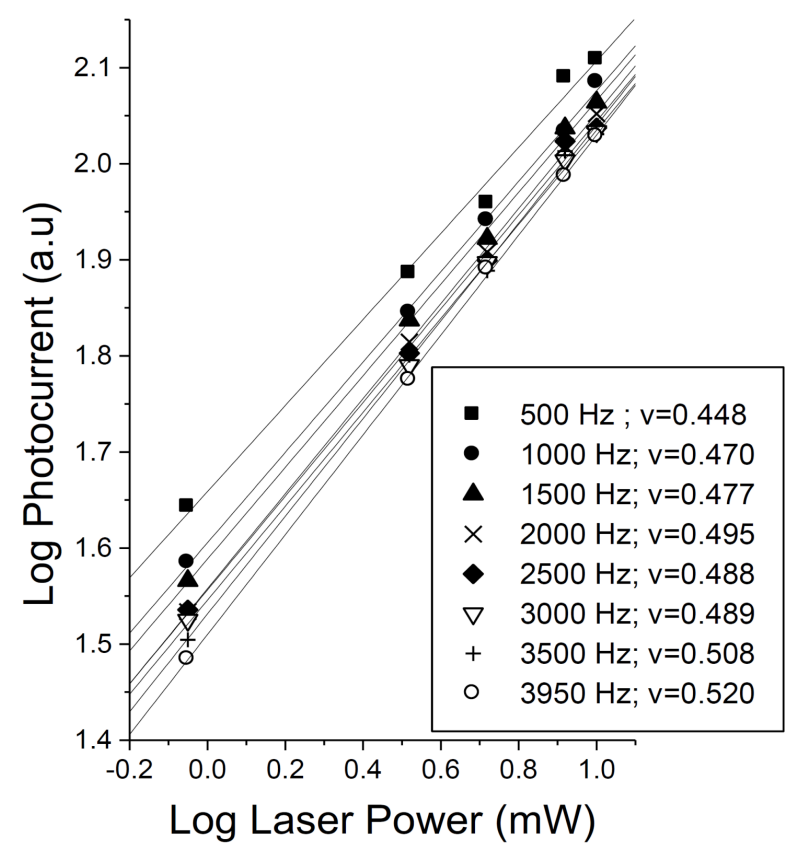

Fig. 6. Modulated photocurrent of a-Se as a function of excitation light intensity at different frequencies.

There are also published results by others, who found $v$ less than 0.5 in field effect structures [18-22]. The high density of states produces high recombination and a poor field profile which inhibits efficient field-aided collection. The low photocurrent causes a high series resistance in the low-field portion of the thin film which degrades the efficiency.

Figure 6 shows the modulated photocurrent of a-Se versus excitation light intensity at different frequencies in the range 500-3950 Hz. It gives almost straight lines. From the slopes of such lines, the calculated $v$ values are illustrated in the figure for each modulation frequency. We also obtained similar results for other thin films of $\mathrm{a}-\mathrm{As}_{2} \mathrm{Se}_{3}$ and $\mathrm{a}-\mathrm{As}_{2} \mathrm{Te}_{3}$ used. Their $v$ values are comparatively shown in Fig. 7, as a function of modulation frequency. The values of $v$ of a- $\mathrm{As}_{2} \mathrm{Se}_{3}$ are larger than those of a-Se, but are smaller than those of a- $\mathrm{As}_{2} \mathrm{Te}_{3}$ for all frequencies measured. On the other hand, the values of $v$ for modulated photocurrent are large when compared their DC equivalences. This is due to the fact that higher lightintensity excitation and modulation frequency produce a larger fraction of photocarriers from deep trapping levels (defects) in the energy gap, and thus these contribute to the photocurrent. The exponent $v$ for DC result must be fit with the $v$ for modulated result extrapolated at zero frequency, but not. This may be due to the DC exponent $v$, which includes some drift or heating effect.

The effects of high and low illuminations on $v$ of a-Se were also examined. This is shown in Fig. 8, in which the high and low intensity ranges are $0.89-10 \mathrm{~mW}$ and $22.25-250 \mu \mathrm{W}$, respectively. Obviously, the value of $v$ almost increases with increasing modulation frequency for both illumination cases. Further, the $v$ is larger for the

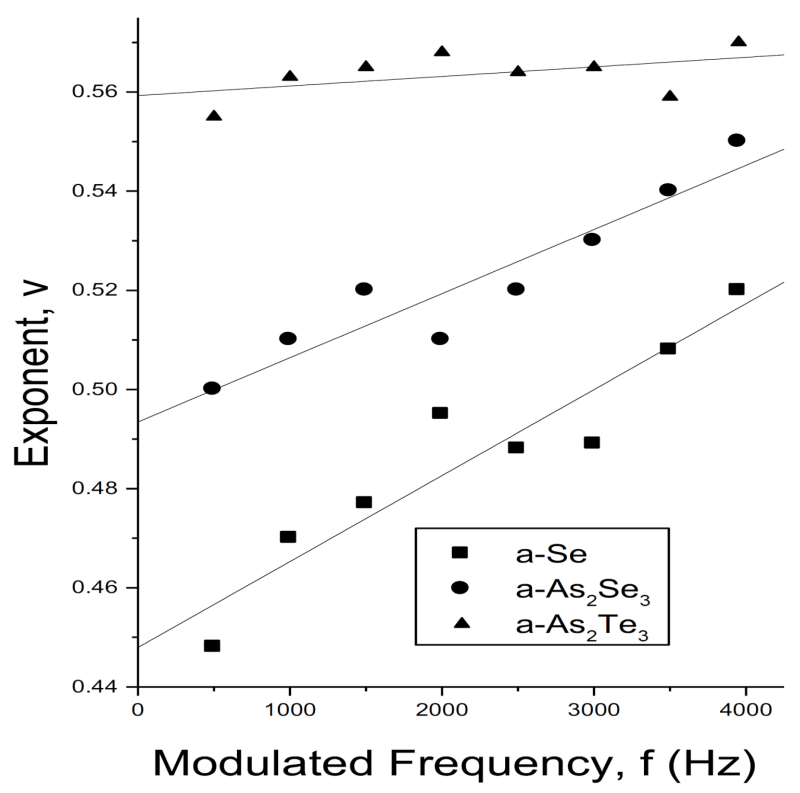

Fig. 7. Comparison of modulated exponent $v$ of a-Se, $\mathrm{a}-\mathrm{As}_{2} \mathrm{Se}_{3}$ and $\mathrm{a}-\mathrm{As}_{2} \mathrm{Te}_{3}$ thin films as a function of modulated frequencies. Lines are drawn as guide for the eye.

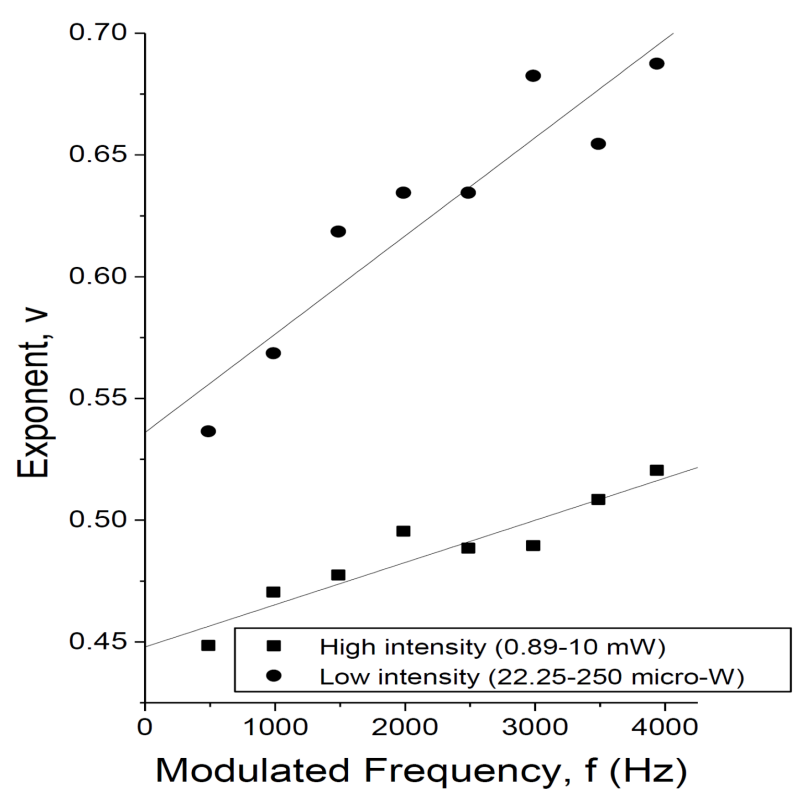

Fig. 8. Comparison of modulated exponent $v$ in a-Se under high and low illuminations. Lines are drawn as guide for the eye.

low illumination intensity than that of high illumination intensity at the same conditions. This is due to that recombination kinetics have dominant effects at low-light intensities rather than trapping. The high intensities create charged defect centers $\left(\mathrm{D}^{+}\right.$and $\left.\mathrm{D}^{-}\right)$in amorphous chalcogenides $[23,24]$. These defects act as recombination centers for photoexcited carriers and thus the number of photocarriers decreases with increasing illumination resulting in a decrease in the net charge. 


\section{Conclusion}

We have performed two sets of experiments covering the resistance in dark and light, and the exponent $v$, the power dependence of the photocurrent with DC and modulated light intensity, on three-types of chalcogenide thin films of a-Se, a- $\mathrm{As}_{2} \mathrm{Se}_{3}$, and $\mathrm{a}-\mathrm{As}_{2} \mathrm{Te}_{3}$. We obtained the dark resistances of 8.85, 9.79, and 5.78 $\mathrm{M} \Omega$ for a-Se, a$\mathrm{As}_{2} \mathrm{Se}_{3}$, and $\mathrm{a}-\mathrm{As}_{2} \mathrm{Te}_{3}$, respectively. The contribution of Te to Se decreases the dark resistance, but the As contribution increases it. This shows that the addition of As to Se creates much more coordination defects in structure. On the other hand, the addition of Te to Se may reduce the energy gap. The decrease in resistance of a-Se with increase in light intensity is due to the decrease in the bulk resistance caused by the increase in photogenerated carriers.

The dependence of $v$ on the applied voltage is very weak, but its magnitude is different for three types of samples. The modulation frequency dependence of $v$ is almost constant for a- $\mathrm{As}_{2} \mathrm{Te}_{3}$, but it increases a little bit for a-Se, and a- $\mathrm{As}_{2} \mathrm{Se}_{3}$ with increasing modulation frequency. However, the values of $v$ are different for three types of samples as in the case of voltage dependence. On the other hand, the value of $v$ is found to be larger at low intensity rather than that at high intensity. However, there is no a complete theoretical model covering the modulation frequency dependence of $v$ in literature. It may be due to the increase of photocarrier release rate with frequency from deeper states, which is thermally activated at room temperatures.

\section{Acknowledgments}

This work was supported by TBAG-2212 (102T091) Contract of The Scientific and Technical Research Council of Turkey (TUBİTAK).

\section{References}

[1] R.A. Street, Hydrogenated Amorphous Silicon, Cambridge University Press, 1991.

[2] N.F. Mott, E.A. Davis, Electronic Processes in NonCrystalline Materials, Clarendon Press, 1971.
[3] Amorphous and Liquid Semiconductors, Ed. J. Tauc, Plenum Press, 1974.

[4] S.R. Elliott, Physics of Amorphous Materials, Longman, 1990.

[5] R.H. Bube, Photoelectric Properties of Semiconductors, Cambridge University Press, 1992.

[6] M.M. El-Nahass, M.H. Ali, E.A.A. El Shazly, I.T. Zedan, Appl. Phys. A 122, 780 (2016).

[7] M.I. Abd-Elrahmann, M.M. Hafiz, A. Qasem, M.A. Abdel-Rahim, Appl. Phys. A 122, 45 (2016).

[8] G.K. Rao, Appl. Phys. A 123, 224 (2017).

[9] M.I. Abd-Elrahman, R.M. Khafagy, S.A. Zaki, M.M. Hafiz, J. Alloys Comp. 571, 118 (2013).

[10] M. Behera, R. Naik, Appl. Phys. A 122, 913 (2016).

[11] K. Shimakawa, A. Kolobov, S.R. Elliott, Adv. Phys. 44, 475 (1995).

[12] M.S. Iovu, S.D. Shutov, M. Popescu, J. Non-Cryst. Solids 299-302, 924 (2002).

[13] P. Krecmer, A.M. Moulin, R.J. Stephenson, J. Raymond, M.E. Welland, S.R. Elliott, Science 277, 1799 (1997).

[14] J.P. de Neufville, S.C. Moss, S.R. Ovshinsky, J. NonCryst. Solids 13, 191 (1974).

[15] R. Kaplan, Solar Energy 84, 401 (2010).

[16] A. Rose, Concepts in Photoconductivity and Allied Problems, Krieger, 1978.

[17] R. Kaplan, Sol. Energy Mater. Sol. Cells 85, 545 (2005).

[18] T. Kagawa, N. Matsumato, K. Kumabe, Phys. Rev. B 28, 4570 (1983).

[19] M. Hack, S. Guha, M. Shur, Phys. Rev. B 30, 6991 (1984).

[20] C. Main, J. Berkin, A. Merazga, in: New Physical Problems in Electronic Materials, Eds. M. Borisov, N. Kirov, J.M. Marshall, A. Vavrek, World Sci., Singapore 1995.

[21] E. Morgado, J. Non-Cryst. Solids 166, 627 (1993).

[22] R.H. Bube, D. Redfield, J. Appl. Phys. 66, 3074 (1989).

[23] A. Ganjoo, Y. Ikeda, K. Shimakawa, J. Non-Cryst. Solids 266-269, 919 (2000).

[24] Z. Zhang, D.A. Drabold, J. Non-Cryst. Solids 241, 195 (1998). 